\title{
RNA interference targeting inhibition of S100A4 suppresses cell growth and promotes apoptosis in human laryngeal carcinoma Hep-2 cells
}

\author{
JIA LIU ${ }^{1}$, SHUANG FU ${ }^{2}$, YINGQI XU ${ }^{1}$ and ZHIHONG ZHENG ${ }^{1,3}$ \\ ${ }^{1}$ Laboratory Animal Center, China Medical University, Shenyang, Liaoning 110001; ${ }^{2}$ Department of Hematology Laboratory, \\ Shengjing Hospital of China Medical University, Shenyang, Liaoning 110022; ${ }^{3}$ Department of Pathology \\ and Pathophysiology Research, China Medical University, Shenyang, Liaoning 110001, P.R. China
}

Received January 7, 2014; Accepted May 9, 2014

DOI: $10.3892 / \mathrm{mmr} .2014 .2345$

\begin{abstract}
S100A4 is a small $\mathrm{Ca}^{2+}$ binding protein that belongs to the S100 family and is involved in a number of cellular functions, including cell cycle control, proliferation, apoptosis, and has a significant role in angiogenesis and neurite extension. However, the exact function and mechanism of S100A4 in laryngeal carcinogenesis remains to be elucidated. The present study was designed to investigate the potential use of RNA interference to inhibit S100A4 expression and activation, as well as the subsequent effect on human laryngeal cancer cell growth and apoptosis. The present study demonstrated that knockdown of S100A4 decreased the proliferation and growth of the human laryngeal cancer Hep-2 cell line. The percentages of the apoptotic cells were $4.23 \pm 1.22,4.92 \pm 1.85$ and $11.70 \pm 4.02 \%$ in the control, negative control and S100A4 short hairpin RNA (shRNA) groups, respectively, indicating significant differences among the different groups. The S100A4-mediated induction of apoptosis was demonstrated to be associated with the activation of caspase- 3 , caspase- 8 and caspase-9. Intratumoral injection of S100A4-shRNA inhibited tumor growth in nude mice. Thus, knockdown of S100A4 inhibited the progression of laryngeal squamous carcinoma, decreased proliferation and promoted apoptosis. S100A4 is a potential candidate for therapeutic targeting of laryngeal carcinoma cells.
\end{abstract}

\section{Introduction}

Head and neck squamous cell carcinoma (HNSCC) is the eighth leading cause of cancer mortality worldwide (1). As one of the most common types of cancer in the head and neck,

Correspondence to: Dr Jia Liu, Laboratory Animal Center, China Medical University, 92 Beier Road, Heping, Shenyang, Liaoning 110001, P.R. China

E-mail:1iu_j@126.com

Key words: S100A4, apoptosis, laryngeal cancer laryngeal squamous carcinoma (LSCC) is a major type of HNSCC (2). The incidence of LSCC in China has been progressively increasing, particularly in the Northeast region. Despite advancements in local control and overall life quality achieved with the use of combined modality therapies, the survival rates for LSCC patients have not improved significantly over the past two decades. With the development of molecular biology, the use of biomarkers in the diagnosis of LSCC in the future and the results from investigating the molecular mechanisms of LSCC may improve treatment and survival rates of LSCC patients (3).

S100A4 is a small $(11.5 \mathrm{kDa})$ acidic calcium binding protein that belongs to the S100 protein family. It has been associated with numerous biological functions, for example, protection of cells from proapoptotic stimuli and stimulation of neurite outgrowth $(4,5)$. Overexpression of S100A4 has been reported in several types of cancer and is correlated with poor patient prognosis (6-8). Previous studies suggested that S100A4 acts as a potential marker of metastasis by regulating cell growth, motility and invasion $(9,10)$. Our previous study revealed that overexpression of S100A4 is associated with the progression of laryngeal cancer, and it is therefore considered an important molecular marker for laryngeal cancer (11). However, the exact role and molecular mechanism of S100A4 in LSCC have not been well characterized. Therefore, in the present study, the biological effects of S100A4 suppression were examined by using the RNA interference method to inhibit S100A4 expression in the laryngeal cancer cell line Hep-2 in order to evaluate the importance of S100A4 and to elucidate the possibilities for utilization of S100A4 in the treatment of LSCC.

\section{Materials and methods}

Cell culture and transfection. The human laryngeal cancer cell line Hep-2 was purchased from the Institute of Biochemistry and Cell Biology, Chinese Academy of Sciences (Shanghai, China) and originated from a metastatic peidermoid carcinoma of the larynx (12). The cell was cultured in RPMI-1640 medium (Invitrogen Life Technologies, Carlsbad, CA, USA) supplemented with $10 \%$ fetal calf serum, $100 \mathrm{U} / \mathrm{ml}$ penicillin and $100 \mathrm{U} / \mathrm{ml}$ streptomycin at $37^{\circ} \mathrm{C}$ in a $5 \% \mathrm{CO}_{2}$ incubator. 
Upon reaching $60-70 \%$ confluence, the cells were divided into two new dish or seeded in six-well plates at a density of $1 \times 10^{5}$ cells per well for further experiments. The Hep-2 cells were transfected with short hairpin RNA (shRNA) control or S100A4 shRNA expression plasmids. Hep-2-shRNA cells expressed an shRNA, specifically targeting S100A4 mRNA, while Hep-2 negative control (Hep-2-shNC) cells expressed an unrelated control sequence. shRNA were transfected into Hep-2 cells using Lipofectamine 2000 (Invitrogen Life Technologies) according to the manufacturer's instructions. Total RNA was prepared $24 \mathrm{~h}$ post transfection and the results of gene knockdown were determined by reverse transcription-quantitative polymerase chain reaction (RT-qPCR). Cells were incubated for $72 \mathrm{~h}$ and then harvested for further experiments.

$R T-q P C R$. The quantification of S100A4 expression levels was performed using an SYBR-Green I-based real-time fluorescence detection method (13). Total RNA was isolated from Hep-2 cells using an mRNA isolation kit (Qiagen, Valencia, CA, USA), and subsequently reverse transcribed using the Reverse Transcription PCR kit kit (Takara Bio Inc., Shiga, Japan) with Oligo-dT primers. cDNA was amplified using an SYBR Premix Ex Taq kit (Takara Bio Inc.) on an ABI 7500 Real-time PCR system (Applied Biosystems, Foster City, CA, USA). GAPDH served as a reference gene and samples without cDNA or without oligonucleotides were used as negative controls. The control GAPDH fragment was amplified using the following primer sequences: GAPDH, forward 5'-ATCATCAGCAATGCCTCC-3' and reverse 5'-CATCACGCCACAGTTTCC 3'; S100A4, forward 5'-CCCTGGATGTGATGGTGTC-3' and reverse 5'-CTCGTTGTCCCTGTTGCTG-3'. The $25 \mu \mathrm{l}$ PCR included $0.5 \mu 1$ cDNA, SYBR ${ }^{\circledR}$ Premix EX Taq ${ }^{\mathrm{TM}} 12.5$ and $2 \mu \mathrm{l}$ of primers. The reaction was run at $95^{\circ} \mathrm{C}$ for $1 \mathrm{~min}$, followed by 45 cycles at $95^{\circ} \mathrm{C}$ for $5 \mathrm{sec}$ and $60^{\circ} \mathrm{C}$ for $20 \mathrm{sec}$. The Ct data was determined using default threshold settings. The threshold cycle $(\mathrm{Ct})$ was defined as the fractional cycle number at which the fluorescence passes the fixed threshold. All measurements were performed at least three times.

Western blot analysis. To determine the levels of protein expression, soluble proteins were isolated using radioimmunoprecipitation assay buffer $(50 \mathrm{mM}$ Tris, $150 \mathrm{mM}$ $\mathrm{NaCl}, 1 \mathrm{mM}$ ethylenediaminetetraacetic acid, 0.2\% NP40) containing complete protease inhibitor followed by centrifugation at $12,000 \mathrm{x} \mathrm{g}$ at $4^{\circ} \mathrm{C}$. The protein concentration was determined from the supernatant using a bicinchoninic acid assay (Beyotime Institute of Biotechnology, Haimen, China) and the results were produced by an ELISA-plate reader (Sunrise RC; Tecan, Theale, UK). Protein extracts were diluted with $5 \mathrm{X}$ sodium dodecyl sulfate (SDS) loading buffer, boiled and resolved on a $12 \% \mathrm{w} / \mathrm{v}$ SDS-PAGE. Protein extract $(40 \mathrm{mg}$ ) was loaded. Following electrophoresis, the proteins were transferred onto a polyvinylidene fluoride membrane (Roche Diagnostics, Mannheim, Germany) by semidry blotting. Detection was performed using rabbit polyclonal anti-S100A4 antibody (Thermo Fisher Scientific, Waltham, MA, USA; 1:1,000) and a mouse monoclonal anti-GAPDH antibody (Santa Cruz Biotechnology, Inc., Santa Cruz, CA,
USA; 1:1,000) was used as a loading control. Secondary antibodies anti-rabbit and anti-mouse IgG coupled to horseradish peroxidase (Beyotime Institute of Biotechnology) were used at dilutions of 1:2,000. Blots were developed with Western Lightning Chemiluminescence Reagent Plus (Thermo Fisher Scientific) and chemiluminescence detection film (Beyotime Institute of Biotechnology).

Cell proliferation assay. S100A4-shRNA Hep-2 cells and control cells between $12 \mathrm{~h}$ and 5 days after transfection, were seeded in a 96-well flat-bottomed plate at a density of $2 \times 10^{3}$ cells in $190 \mu \mathrm{l}$ of the medium per well. Cell proliferation was determined using the Cell Counting kit-8 (CCK-8; Beyotime Institute of Biotechnology) according to the manufacturer's instructions. After $10 \mu \mathrm{l}$ CCK- 8 was added to each well, the cells were incubated at $37^{\circ} \mathrm{C}$ for $2 \mathrm{~h}$ and the absorbance was monitored using a microplate reader (Sunrise RC) at a wavelength of $490 \mathrm{~nm}$. Each experiment was performed in triplicate and repeated three times. Data are presented as the mean \pm standard deviation.

Anchorage-independent growth assay. The anchorage-independent growth of cells was determined by assaying colony formation in soft agar. Following transfection, single cell suspension of transfected Hep-2 cells was prepared by trypsinization and homogenization. Cells were plated onto each well of a six-well plate at a density of 1,000 cells/well in RPMI-1640 containing $10 \%$ fetal bovine serum and $0.3 \%$ low melting point agarose (Amresco, Solon, OH, USA) on a base layer of $0.6 \%$ low melting point agarose. The number of colonies $>50 \mu \mathrm{m}$ was counted and images were captured following 2 weeks of incubation at $37^{\circ} \mathrm{C}$. The experiments were performed three times independently.

Apoptosis assay. The Hep-2-shRNA transfected cells and control cells were harvested, fixed and resuspended in phosphate-buffered saline. Apoptotic cells were measured using an annexin V-phycoerythrin (PE)/7-aminoactinomycin D (7AAD) kit (Nanjing KeyGen Biotech., Co., Ltd., Nanjing, China) according to the manufacturer's instructions. Briefly, annexin V-PE and 7AAD were added to a mixture containing $100 \mu 1$ of cell resuspension in binding buffer (BD Biosciences, Franklin Lakes, NJ, USA). The cells were vortexed and incubated for $15 \mathrm{~min}$ at room temperature in the dark and $400 \mu \mathrm{l}$ of binding buffer was added to the mixture for flow cytometric analysis using a Becton Dickinson FACScan Flow Cytometer (FACScan; Becton Dickinson, Franklin Lakes, NJ, USA).

Caspase $-3,-8$ and -9 activity assay. The activity of caspase- 3 , -8 and -9 was determined using the caspase $-3,-8$ and -9 activity kit (Beyotime Institute of Biotechnology). To evaluate the activity of caspase-3, -8 and -9, Hep-2-shRNA transfected and control Hep- 2 cells were homogenized in $100 \mathrm{ml}$ reaction buffer [1\% NP-40, 20 mM Tris- $\mathrm{HCl}$ (pH 7.5), 137 mM Nicotinamide adenine dinucleotide and $10 \%$ glycerol] containing $10 \mathrm{ml}$ caspase-3, - 8 and -9 substrate (Ac-DEVD-pNA; $2 \mathrm{mM}$ ) following all treatments. Lysates were incubated at $37^{\circ} \mathrm{C}$ for $2 \mathrm{~h}$. Samples were measured with an ELISA reader (Sunrise $\mathrm{RC}$ ) at an absorbance of $405 \mathrm{~nm}$. 
Subcutaneous xenografts in nude mice. All the animals (Vital River Laboratory Animal Technology Co., Ltd., Beijing, China) in the present study were housed in a pathogen-free facility and the animal experiments were performed in accordance with the institutional animal welfare guideline of The Center for Laboratory Animal Research, China Medical University (Shenyang, Liaoning, China). Cells were injected subcutaneously into BALB/c nude mice (6-8 weeks). The tumor volume (TV) was calculated using the following formula: TV $\left(\mathrm{mm}^{3}\right)=\left(\right.$ Length $\left.\mathrm{x} \mathrm{Width}^{2}\right) / 2$.

Statistical analysis. Unless otherwise stated, each experiment was performed a minimum of three times. Data were subjected to statistical analysis using Statistical Package for the Social Sciences software (version 13.0; SPSS, Inc., Chicago, IL, USA) and presented as the mean \pm standard error of the mean. The independent Student's t-test or analysis of variance was used to compare the continuous variables among groups. The Kaplan-Meier estimate was used for survival analysis. $\mathrm{P}<0.05$ was considered to indicate a statistically significant difference.

\section{Results}

Expression of S100A4 following shRNA transfection in Hep-2 cells. The mRNA and protein expression of S100A4 in cultured Hep-2 cells $72 \mathrm{~h}$ after shRNA transfection was detected by RT-qPCR and western blot analysis. Hep-2-shNC cells were also transfected as a control (Fig. 1A). The results of the analysis of S100A4 are shown in Fig. 1. Following transfection with S100A4 shRNA, the Hep-2 cells showed significant downregulation of the $S 100 A 4$ gene at the mRNA and protein levels (Fig. 1B and $\mathrm{C} ; \mathrm{P}<0.05$ ).

Downregulation of S100A4 expression decreases the proliferation of Hep-2 cells. As determined by the CCK-8 assay, inhibition of S100A4 in Hep-2 cells resulted in a significant decrease in cellular proliferation at 2, 4, 6 and 8 days after transfection $(\mathrm{P}<0.05)$. The results indicated that S100A4 suppression correlated with decreased proliferation in Hep-2 cells (Fig. 2A).

Effect of S100A4 inhibition on anchorage-independent growth. The anchorage-independent growth of cells was determined by assaying colony formation in soft agar. Our results demonstrated that, compared with Hep-2-shNC cells, the colonies in the Hep-2-shRNA cells were significantly decreased in number and notably smaller in size (Fig. 2B and C).

Downregulation of S100A4 expression increases apoptosis of Hep-2 cells. Apoptosis of Hep-2-shNC and Hep-2-shRNA cells was examined by flow cytometry. As shown in Fig. 3, after 3 days, 4.4 and $4.92 \%$ of the control cells and Hep-2-shNC cells were apoptotic, while $11.7 \%$ of Hep-2-shRNA cells were apoptotic.

Downregulation of S100A4 increases the activity of caspase-3, -8 and -9. Caspase proteins are cysteine proteases that act downstream of the B-cell lymphoma 2 family by initiating cellular breakdown during apoptosis. Among the effector caspases, caspase- 3 is the most frequently involved in apoptosis. To

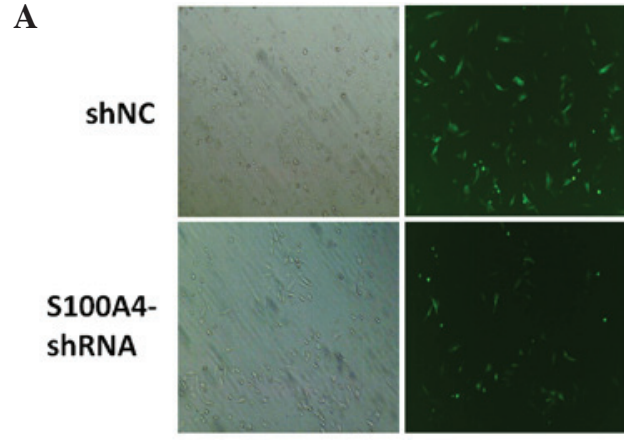

B
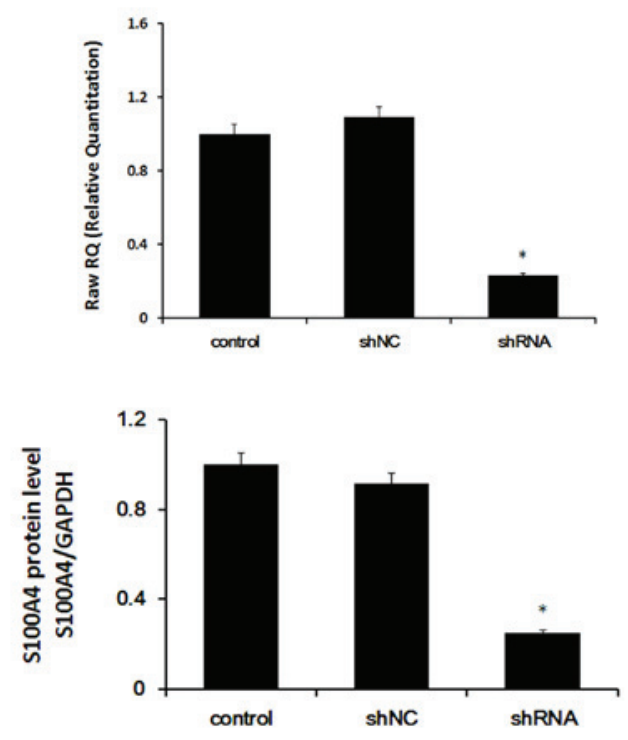

C

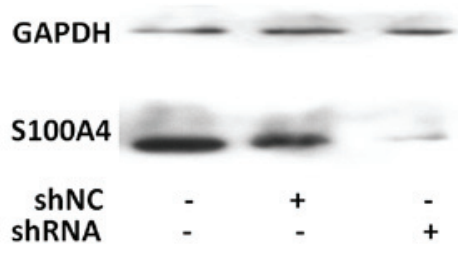

Figure 1. Knockdown of S100A4 mRNA and protein expression in Hep-2 cells. (A) Hep-2 cells transfected with S100A4 shRNA or S100A4 shNC were grown under normal culture conditions and the cells were revealed by contrast and fluorescence microscopy under the same phase. RNA and protein were detected by (B) reverse transcription-quantitative polymerase chain reaction and $(\mathrm{C})$ western blotting, respectively. GAPDH was used as an internal control. ${ }^{*} \mathrm{P}<0.05$, compared with the control group. shRNA, short hairpin RNA; NC, negative control.

determine whether caspase- $3,-8$ and -9 are activated following S100A4 shRNA treatment, caspase-3, -8 and -9 activity was measured by the caspase $-3,-8$ and -9 activity kit. In the present study, the results demonstrated that compared with the control cells, treatment of cells with S100A4 shRNA caused a significant increase in caspase-3, caspase- 8 and caspase- 9 activation $(\mathrm{P}<0.05)$. The activity of caspase- 3 , caspase- 8 and caspase- 9 in cells treated with S100A4 shRNA increased $>12 \%(\mathrm{P}<0.05)$, compared with cells treated with control shRNA (Fig. 4). These results suggested that the downregulation of S100A4 was able to significantly increase the activity of caspase-3, caspase- 8 and caspase- 9 in Hep- 2 cells

Intratumoral injection of S100A4 suppresses the development of human Hep-2 cells in nude mice. Since inhibition of S100A4 
A

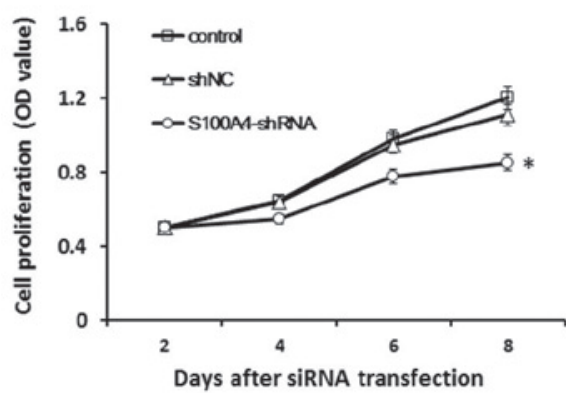

B

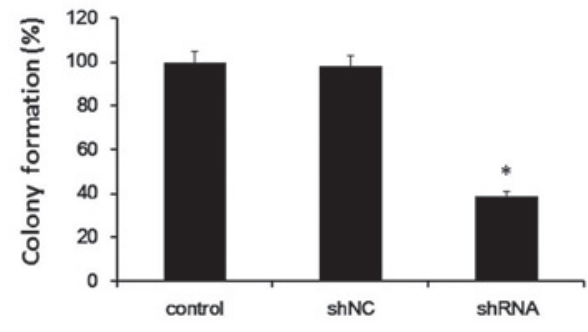

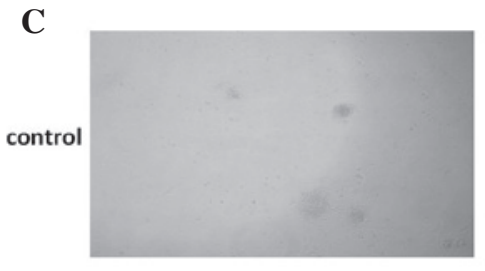
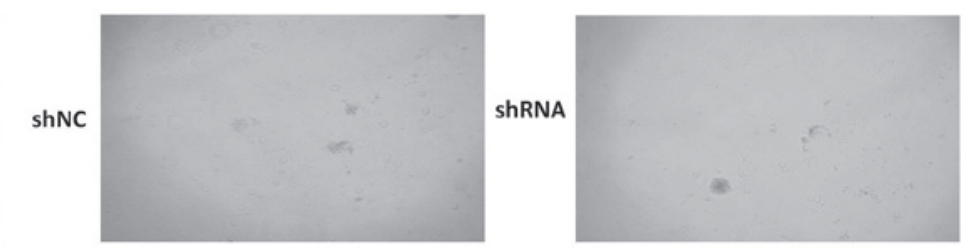

Figure 2. Downregulation of S100A4 expression decreases the proliferation of Hep-2 cells. (A) Growth curves of Hep-2 cells transfected with Hep-2-shNC, Hep-2-shRNA and the control group, indicating that S100A4 downregulation significantly inhibited Hep-2 cell growth. (B and C) Number of colonies in Hep-2-shRNA and Hep-2-shNC cells is expressed as a percentage of the number of colonies in the control cells. Each experiment was performed in triplicate. Bars indicate standard deviation. ${ }^{*} \mathrm{P}<0.05$, compared with the control group. shRNA, short hairpin RNA; NC, negative control.

A

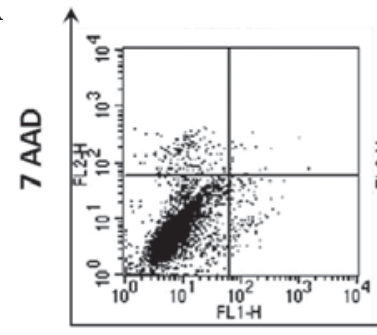



Annexin V-PE

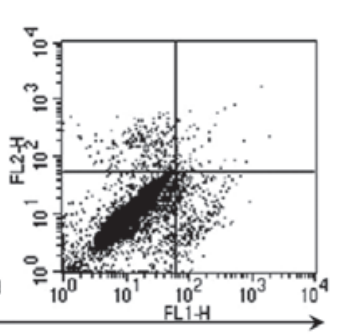

B

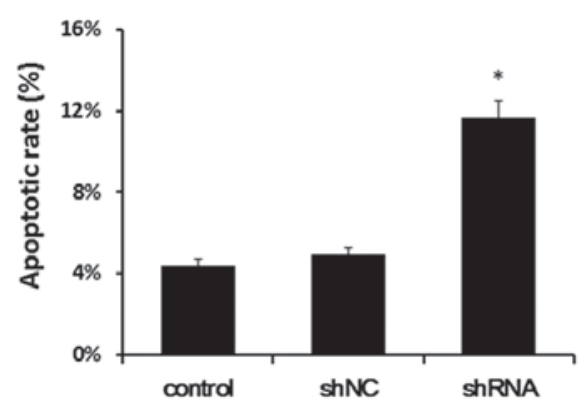

Figure 3. Effect of S100A4 on Hep-2 cell apoptosis measured by flow cytometry. (A) Diagrams of flow cytometry of Hep-2 cells stained with and treated with Hep-2-shRNA, Hep-2-shNC and control are shown, respectively. (B) Data indicated that the number of apoptotic cells transfected with Hep-2-shRNA was significantly greater than that in the control groups. " $\mathrm{P}<0.05$, compared with the control group. shRNA, short hairpin RNA; NC, negative control; PE, phycoerythrin; 7AAD, 7-aminoactinomycin D.

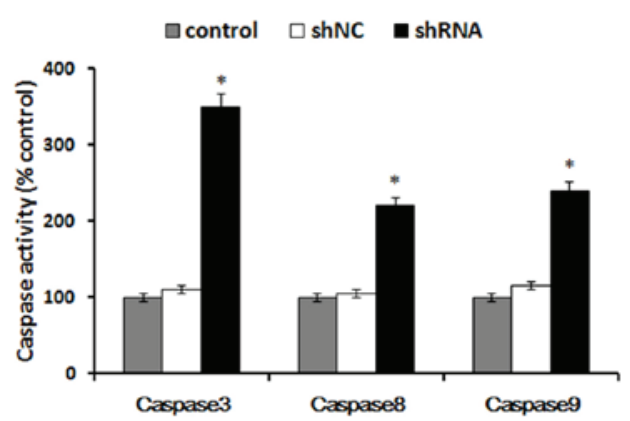

Figure 4. Detection of caspase-3, -8 and -9 activities in S100A4 knockdown Hep- 2 cells. The activities of caspase- $3,-8$ and -9 were detected. Treatment with S100A4 shRNA increases the activity of caspase-3, -8 and -9 in Hep-2 cells. ${ }^{~} \mathrm{P}<0.05$, versus the control group. shRNA, short hairpin RNA; NC, negative control.

reduced Hep-2 cell proliferation in vitro, whether these in vitro data have in vivo relevance was examined. To accomplish this goal, Hep- 2 cells generated tumors when $2 \times 10^{5}$ cells were injected into the flank of nude mice. Visible tumors had developed at the injection sites within 2 weeks (Fig. 5A and B). The S100A4 knockdown Hep-2 cells significantly reduced the tumor volumes and prolonged the survival rate of nude mice (Fig. $5 \mathrm{C}$ and D). Our data indicated that downregulation of S100A4 decreased the tumorigenicity of laryngeal cancer in vivo.

\section{Discussion}

Previous studies have demonstrated that S100A4 expression is correlated with a poor prognosis in cancer patients $(8,14,15)$. S100A4 is involved in tumor metastasis by affecting cell growth, invasion and motility $(16,17)$. Our previous study reported a significant correlation between S100A4 expression levels and LSCC (11). However, the role of S100A4 in laryngeal cancer remains to be elucidated. To address the question of whether S100A4 was able to serve as a therapeutic target for laryngeal cancer, the RNA interference method was employed in an attempt to reduce the expression of S100A4 in cultured Hep-2 cells. As shown in Fig. 1, significant suppression of S100A4 expression was observed using RT-qPCR and western blotting. 
A

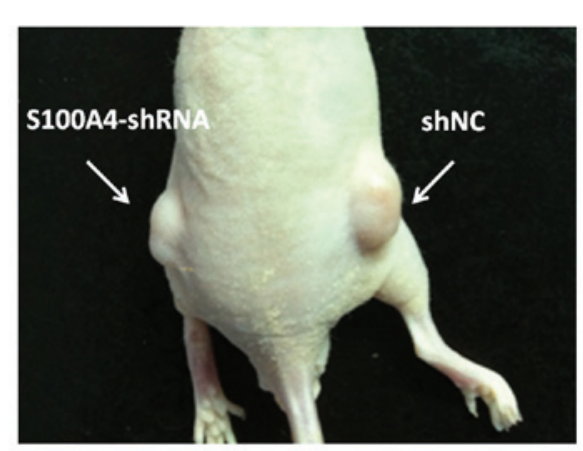

C

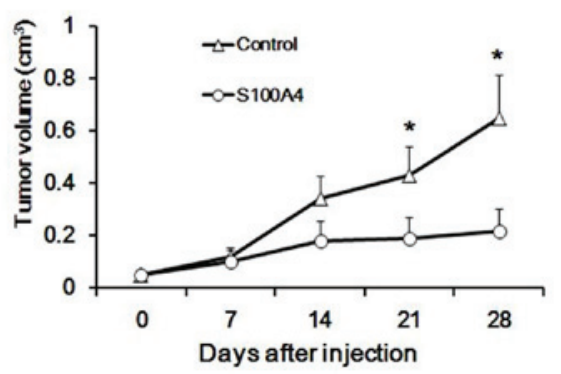

B

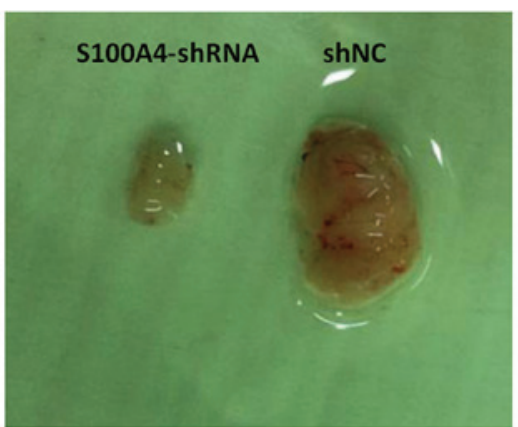

D



Figure 5. Knockdown of S100A4 inhibits laryngeal cancer xenograft tumor growth. (A and B) Tumor growth of control Hep-2 cells or S100A4-knockdown Hep-2 cells. (C) Tumors were measured at a regular interval for up to 28 days with calipers and the tumor volume was calculated. (D) Survival curves of tumor-bearing nude mice treated with the S100A4 shRNA and control were recorded. shRNA, short hairpin RNA; NC, negative control.

S100A4 is important in a variety of cellular events, including cell growth and apoptosis $(18,19)$. However, previous discrepancy studies suggest the effect of S100A4 on cell growth and apoptosis depends on the cell type. For instance, the suppression of S100A4 reduces the proliferative potential and induction of apoptosis in certain cancer cells, including pancreatic cancer cells (20) and gastric cancer cells (21) in vitro and in vivo. Whereas another study demonstrated that in human osteosarcoma cells, in vitro cell proliferation was unaltered by anti-S100A4 ribozyme (22). Compared with anti-S100A4 ribozyme transfected counterpart II-11b cells, the S100A4-secreting human osteosarcoma cell line OHS was more sensitive to IFN- $\gamma$-mediated apoptosis in a previous study, and the S100A4/IFN- $\gamma$-mediated induction of apoptosis was demonstrated to be independent of caspase activation (23). The in vitro growth suppression of laryngeal cancer Hep-2 cells was analyzed. The present study demonstrated that the growth of Hep-2 cells was inhibited by transfection with S100A4 shRNA. Furthermore, anchorage-independent growth refers to the ability of cells to survive and proliferate in the absence of attachment to the extracellular matrix, which is modeled in vitro by colony formation in soft agar. Our data demonstrated that, compared with shNC and control group cells, colony formation of Hep-2-shRNA cells was profoundly suppressed.

To elucidate the growth and colony formation suppression, FACS analyses was performed 3 days after transfection of the shRNA and the results indicated that the shRNA-mediated knockdown of S100A4 led to apoptosis of the laryngeal cancer cells. It was hypothesized that the increased apoptosis in Hep-2 cells transfected with S100A4-shRNA was able to contribute to the reduction of cell numbers. Previous studies demonstrated that S100A4 interacts with the tumor suppressor p53 to affect cell growth and p53-dependent apoptosis (24). It was suggested that the control functions of p53 will be abrogated at the G1-S checkpoint when the complex of p53 with S100A4 and the sequestration of p53 stimulate the cell enter to the $\mathrm{S}$ phase. The p53-dependent transactivation target genes were related with the state of apoptosis. S100A4 knockdown leads to p53-dependent cell cycle arrest and increased cisplatin-induced apoptosis (25).

Apoptosis is a type of cell death that may occur in multicellular organisms. It represents highly programmed mechanisms by activated proteolytic caspases. Exploration of the underlying mechanisms of apoptosis by elucidating the patterns of these factors in the apoptosis may be critical. It is understood that caspase- 8 is important in transduction of the death-receptor pathway while caspase- 9 is involved in the mitochondrial pathway (26). Once activated, caspase- 8 and caspase-9 activate downstream caspase-3, which is a key executioner caspase for triggering cell apoptosis (27). However, a previous study suggested that caspase- 8 is not always activated early in the context of Fas signaling, in certain cells, caspase- 3 in turn activates caspase-6, which was revealed to be required for the activation of downstream caspase-8 (28). The present study demonstrated that the activity of caspase-3, -8 and -9 was higher in response to S100A4-shRNA, suggesting that apoptosis proceeded at least via the mitochondrial pathway. Overall, future research in order to delineate the details of how S100A4 is involved in apoptosis is required.

Following determining the functions of S100A4 in Hep-2 cells in vitro, the present study next sought to determine whether downregulation of S100A4 expression reduces the tumor-forming ability of Hep-2 cells in vivo. The present study 
aimed to address this question by using S100A4 shRNA in a xenograft laryngeal cancer model. Knockdown of S100A4 in Hep-2 cells significantly reduced the tumor volumes and prolonged the survival rate of nude mice. Shi et al (29) obtained similar results in which S100A4 knockdown was able to inhibit the formation and growth of thyroid cancer. Lo et al (30) identified that knockdown of S100A4 affected epithelial-mesenchymal transition-calcium-embryonic stem cells associated genes, including TP53, Notch2, phosphatase and tensin homolog (PTEN) and phosphoinositide 3-kinase (PI3K). The Notch and PTEN/PI3K/Akt signaling pathways have been demonstrated to regulate the tumorigenicity of cancer (30). All these results suggest the importance of aberrant expression of S100A4 in the cancer process and intratumoral injections of S100A4-shRNA exert a strong antitumor effect in vivo.

In conclusion, the present study demonstrated that inhibition of S100A4 through RNA interference resulted in significantly decreased cell proliferation and increased apoptosis in laryngeal cancer in vitro and in vivo. The present study also provided evidence that S100A4 promoted apoptosis through released caspase-3, -8 and -9 . Whether apoptosis occurred via the mitochondrial pathway or the death receptor-mediated pathway and which factors were involved in this process requires further investigation. The present study showed that S100A4 exhibits a major role in Hep-2 cell proliferation and apoptosis and targeting of the S100A4 signaling may be a potential therapeutic target for laryngeal cancer.

\section{Acknowledgements}

The present study was supported by the National Natural Science Foundation of China (grant no. 81301766).

\section{References}

1. Ragin CC, Modugno F and Gollin SM: The epidemiology and risk factors of head and neck cancer: a focus on human papillomavirus. J Dent Res 86: 104-114, 2007.

2. Jemal A, Siegel R, Ward E, Murray T, Xu J and Thun MJ: Cancer statistics, 2007. CA Cancer J Clin 57: 43-66, 2007.

3. Marioni G, Marchese-Ragona R, Cartei G, Marchese F and Staffieri A: Current opinion in diagnosis and treatment of laryngeal carcinoma. Cancer Treat Rev 32: 504-515, 2006.

4. Mahon PC, Baril P, Bhakta V, et al: S100A4 contributes to the suppression of BNIP3 expression, chemoresistance, and inhibition of apoptosis in pancreatic cancer. Cancer Res 67: 6786-6795, 2007.

5. Pedersen MV, Køhler LB, Grigorian M, et al: The Mts1/S100A4 protein is a neuroprotectant. J Neurosci Res 77: 777-786, 2004

6. Sekine H, Chen N, Sato K, et al: S100A4, frequently overexpressed in various human cancers, accelerates cell motility in pancreatic cancer cells. Biochem Biophys Res Commun 429: 214-219, 2012.

7. Boye K, Nesland JM, Sandstad B, Haugland Haugen M, Mælandsmo GM and Flatmark K: EMMPRIN is associated with S100A4 and predicts patient outcome in colorectal cancer. $\mathrm{Br}$ J Cancer 107: 667-674, 2012.

8. Li H, Liu Z, Xu C, et al: Overexpression of S100A4 is closely associated with the progression and prognosis of gastric cancer in young patients. Oncol Lett 5: 1485-1490, 2013

9. Klingelhöfer J, Grum-Schwensen B, Beck MK, et al: Anti-S100A4 antibody suppresses metastasis formation by blocking stroma cell invasion. Neoplasia 14: 1260-1268, 2012.

10. Wang $Z$ and Griffin $M$ : The role of TG2 in regulating S100A4-mediated mammary tumour cell migration. PLoS One 8: e57017, 2013
11. Liu J, Guo Y, Fu S, Yang M, Sun KL and Fu WN: Hypomethylation-induced expression of S100A4 increases the invasiveness of laryngeal squamous cell carcinoma. Oncol Rep 23: 1101-1107, 2010.

12. Toolan HW: Transplantable human neoplasms maintained in cortisone-treated laboratory animals: H.S. No. 1; H.Ep. No. 1; H.Ep. No. 2; H.Ep. No. 3; and H.Emb.Rh. No. 1. Cancer Res 14: 660-666, 1954

13. Boehm D, Herold S, Kuechler A, Liehr T and Laccone F: Rapid detection of subtelomeric deletion/duplication by novel real-time quantitative PCR using SYBR-green dye. Hum Mutat 23: 368-378, 2004.

14. Zhao Y,Zhang T and Wang Q: S100 calcium-binding protein A4 is a novel independent prognostic factor for the poor prognosis of gastric carcinomas. Oncol Rep 30: 111-118, 2013.

15. Roh J, Knight S, Chung JY, et al: S100A4 expression is a prognostic indicator in small intestine adenocarcinoma. J Clin Pathol 67: 216-221, 2014

16. Berge G, Pettersen S, Grotterød I, Bettum IJ, Boye K and Mælandsmo GM: Osteopontin - an important downstream effector of S100A4-mediated invasion and metastasis. Int J Cancer 129: 780-790, 2011.

17. Sack U, Walther W, Scudiero D, et al: S100A4-induced cell motility and metastasis is restricted by the Wnt/beta-catenin pathway inhibitor calcimycin in colon cancer cells. Mol Biol Cell 22: 3344-3354, 2011.

18. Chen X, Luther G, Zhang W, et al: The E-F hand calcium-binding protein S100A4 regulates the proliferation, survival and differentiation potential of human osteosarcoma cells. Cell Physiol Biochem 32: 1083-1096, 2013.

19. Yang XC, Wang X, Luo L, et al: RNA interference suppression of A100A4 reduces the growth and metastatic phenotype of human renal cancer cells via NF-kB-dependent MMP-2 and bcl-2 pathway. Eur Rev Med Pharmacol Sci 17: 1669-1680, 2013.

20. Tabata T, Tsukamoto N, Fooladi AA, et al: RNA interference targeting against S100A4 suppresses cell growth and motility and induces apoptosis in human pancreatic cancer cells. Biochem Biophys Res Commun 390: 475-480, 2009.

21. Hua J, Chen D, Fu H, et al: Short hairpin RNA-mediated inhibition of S100A4 promotes apoptosis and suppresses proliferation of BGC823 gastric cancer cells in vitro and in vivo. Cancer Lett 292: 41-47, 2010.

22. Maelandsmo GM, Hovig E, Skrede M, et al: Reversal of the in vivo metastatic phenotype of human tumor cells by an anti-CAPL (mts1) ribozyme. Cancer Res 56: 5490-5498, 1996.

23. Pedersen KB, Andersen K, Fodstad $\varnothing$ and Maelandsmo GM: Sensitization of interferon-gamma induced apoptosis in human osteosarcoma cells by extracellular S100A4. BMC Cancer 4: $52,2004$.

24. Grigorian M, Andresen S, Tulchinsky E, et al: Tumor suppressor p53 protein is a new target for the metastasis-associated Mts1/S100A4 protein: functional consequences of their interaction. J Biol Chem 276: 22699-22708, 2001.

25. Orre LM, Panizza E, Kaminskyy VO, et al: S100A4 interacts with p53 in the nucleus and promotes p53 degradation. Oncogene 32: 5531-5540, 2013.

26. Cho SG and Choi EJ: Apoptotic signaling pathways: caspases and stress-activated protein kinases. J Biochem Mol Biol 35: 24-27, 2002.

27. Fan TJ, Han LH, Cong RS and Liang J: Caspase family proteases and apoptosis. Acta Biochim Biophys Sin (Shanghai) 37: 719-727, 2005.

28. Slee EA, Harte MT, Kluck RM, et al: Ordering the cytochrome c-initiated caspase cascade: hierarchical activation of caspases-2, -3, -6, -7, -8, and -10 in a caspase-9-dependent manner. J Cell Biol 144: 281-292, 1999.

29. Shi Y, Zou M, Collison K, et al: Ribonucleic acid interference targeting S100A4 (Mts1) suppresses tumor growth and metastasis of anaplastic thyroid carcinoma in a mouse model. J Clin Endocrinol Metab 91: 2373-2379, 2006.

30. Lo JF, Yu CC, Chiou SH, et al: The epithelial-mesenchymal transition mediator S100A4 maintains cancer-initiating cells in head and neck cancers. Cancer Res 71: 1912-1923, 2011. 